\title{
EFEITO DO CULTIVO CONTÍNUO DA CANA-DE-AÇÚCAR EM PROPRIEDADES FISICAS DE UM LATOSSOLO VERMELHO ESCURO ${ }^{1}$
}

\author{
J.C.M. de OLIVEIRA ${ }^{2,5}$; C.M.P. VAZ OK.REICHARDT $^{2,4,6}$ \\ ${ }^{2}$ Centro de Energia Nuclear na Agricultura/USP, C.P. 96, CEP: 13400-970, Piracicaba,SP \\ ${ }^{3}$ CNPDIA/EMBRAPA, C.P. 741, CEP: 13560-970, São Carlos,SP \\ 'Departamento de Física e Meteorologia-ESALQ/USP, C.P. 9, CEP: 13418-900, Piracicaba,SP \\ ${ }^{5}$ Bolsista da FAPESP \\ ${ }^{6}$ Bolsista do $\mathrm{CNPq}$
}

\begin{abstract}
RESUMO: Com o objetivo de estudar as caracteristicas físicas do solo, sob monocultura, o presente trabalho avaliou a distribuiçao de tamanho de partículas, presença de camadas compactadas, porosidade total, macro e microporosidade de um latossolo vermelho escuro cultivado com cana-de-açúcar. As observações foram feitas em 1992-1993 utilizando amostras de solo sob vegetação natural de floresta e sob cultivo contínuo por 16, 30 e 50 anos. Amostras foram coletadas em camadas de $10 \mathrm{~cm}$ até a profundidade de $1 \mathrm{~m}$. Os resultados indicam variações significativas do teor de argila dos borizontes superficiais em função do tempo de cultivo e bem como aumentos da densidade do solo, para as parcelas cultivadas. Variaçōes também foram encontradas para a macro e microporosidade, apenas para os horizontes submetidos às práticas de cultivo.
\end{abstract}

Descritores: cultivo contínuo, monocultura, distribuição de partículas, cana-de-açúcar, latossolo vermelho escuro

\section{EFFECT OF CONTINUOUS SUGAR-CANE CULTIVATION ON PHYSICAL SOIL PROPERTIES OF A DARK RED LATOSOL}

\begin{abstract}
With the objetive of studying soil physical characteristics of a dark red latosol under Iong term cultivation of sugar-cane monoculture, the present paper evaluated soil particle size distributions, soil density and porosity of soil samples from plots cultivated for 16,30 and 50 years to sugar-cane as compared with soil samples from natural rain-forest. Results indicated significant changes in clay contents and soil density of the surface horizon, as a function of soil use. Changes were also observed in micro and macroporosities of these samples. Key Words: continuous cropping, monoculture, particle size distribution, sugar-cane, dark red latosol
\end{abstract}

\section{INTRODUÇÃO}

A substituição de florestas por culturas agrícolas causa, invariavelmente, mudanças nas características físicas e químicas do solo que, em muitos casos, levam a uma degradação e, consequentemente, perdas na produtividade do sistema de produção agrícola.

No caso da cultura da cana-de-açúcar no Estado de São Paulo, áreas cobertas por mata natural foram gradativamente substituídas por canaviais e têm sido mantidas com monocultura por períodos de até 60 anos. Esta substituição, em conjunto com cultivo contínuo, prolongado e, mais recentemente, intensivo, pode causar mudanças nas características físicas do solo, sendo frequentes as reduções da porosidade total e da macroporosidade e aumentos da densidade do solo e da microporosidade (SILVA, 1980; WOOD, 1985; CINTRA \& CUNHA, 1987; SILVA \& RIBEIRO, 1992; BORGES, 1993).

O presente trabalho teve como objetivo avaliar o grau de modificação de algumas características físicas e morfológicas de um solo submetidos ao cultivo prolongado com cana-deaçúcar.

\footnotetext{
(1) Parte da tese de doutorado do primeiro autor, junto ao Curso de Pós-Graduação do CENA/USP, Piracicaba,(SP).
} 


\section{MATERIAL E MÉTODOS}

O experimento situou-se no município de Piracicaba,SP $\left(22^{\circ} 43^{\prime} \mathrm{S} ; 4^{\circ} 38^{\prime} \mathrm{W}\right)$, em área da Usina Capuava S/A.

O solo, descrito por CERRI et al. (1991), é um latossolo vermelho escuro, desenvolvido sobre intercalações de arenito basáltico. A área escolhida é caracterizada por um relevo plano com declividades menores que $1 \%$ e os pontos de coleta foram distantes entre si de não mais que $700 \mathrm{~m}$. Foram escolhidas quatro situações distintas de uso da terra: uma coberta por vegetação nativa (floresta latifoliada tropical) e as outras três desmatadas e cultivadas continuamente com cana-de-açúcar durante dezesseis, trinta e cinquenta anos.

A parcela cultivada por cinquenta anos foi desmatada manualmente. As primeiras arações e gradagens foram feitas com tração animal, sendo que, atualmente, as gradagens são feitas com tração mecânica. As parcelas de trinta e de dezesseis anos foram desmatadas mecanicamente, sendo que todas as gradagens foram feitas com tração mecânica.

Em cada uma das áreas foi aberta uma trincheira, sendo as amostras coletadas de $10 \mathrm{em}$ $10 \mathrm{~cm}$ ate $100 \mathrm{~cm}$ de profundidade. Foram colhidas amostras com estrutura deformada, para análise granulométrica e, com estrutura indeformada (cilindros de $50 \mathrm{~cm}^{3}$ ) para a determinação da porosidade total, macro e microporosidade e, ainda, amostras na forma de torróes, para determinação da densidade do solo.
Para a análise granulométrica de amostras de terra, foi utilizado o método de atenuação de radiação gama (VAZ et al., 1992; OLIVEIRA, 1994) e os resultados apresentados de acordo com a Escala do Departamento de Agricultura dos Estados -Unidos. A densidade do solo foi determinada utilizando-se uma técnica alternativa, a tomografia computadorizada (CRESTANA et al., 1992; VAZ et al., 1992); a porosidade total foi determinada utilizando-se os valores da densidade do solo e densidade de partículas (KIEHL, 1979); para a microporosidade, utilizou-se o método da desorção de água em mesa de tensão, aplicando-se tensão correspondente a uma altura de coluna de água de $60 \mathrm{~cm}$; a macroporosidade foi calculada pela diferença entre a porosidade total e a microporosidade. Todos os dados referem-se às médias de três repetições por profundidade.

\section{RESULTADOS E DISCUSSÃo}

A discussão dos resultados pressupõe tratar-se de uma área homogênea de um mesmo solo, avaliada de forma tradicional por CERRI et al. (1991). Devido ao tipo de amostragem feita (uma trincheira por parcela) não foi possível incluir na análise a variabilidade espacial natural do solo.

Os resultados da análise granulométrica de amostras de terra são mostrados na figura 1 . Observa-se na figura 1a, que a porção superficial do solo sob floresta possui quantidades de argila bem maiores $(50-60 \%)$ que a dos três perfís cultivados $(30-40 \%)$.

TABELA 1 - Valores médios das porcentagens de argila, silte e areia total, nos intervalos de 0 a 100,0 a 60 e 60 a $100 \mathrm{~cm}$ de profundidade.

\begin{tabular}{cccccccccc}
\hline \hline Área & \multicolumn{2}{c}{$\begin{array}{c}\mathrm{I} / \Delta \mathrm{z} \\
\end{array}$} & \multicolumn{2}{c}{$0-100 \mathrm{~cm}$} & & \multicolumn{3}{c}{$\mathrm{I} / \Delta \mathrm{z}$} & \multicolumn{3}{c}{$\mathrm{c} \mathrm{cm}$} & \multicolumn{3}{c}{$60-100 \mathrm{~cm}$} \\
\hline & Argila & Silte & Areia & Argila & Silte & Areia & Argila & Silte & Areia \\
Floresta & 65 & 13 & 21 & 64 & 14 & 20 & 65 & 11 & 21 \\
16 anos & 58 & 16 & 27 & 57 & 17 & 31 & 68 & 13 & 20 \\
30 anos & 59 & 18 & 21 & 55 & 21 & 23 & 66 & 13 & 19 \\
50 anos & 57 & 16 & 27 & 51 & 18 & 31 & 65 & 13 & 22 \\
\hline \hline
\end{tabular}


Calculando-se a razão:

$$
\frac{I_{z_{1}}^{z_{2}}}{z_{2}-z_{1}}
$$

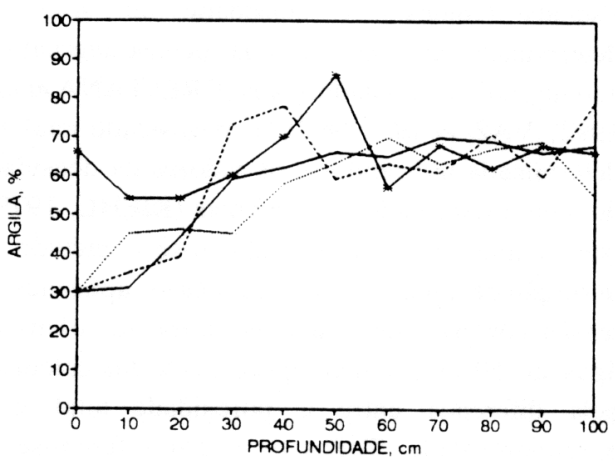

b)

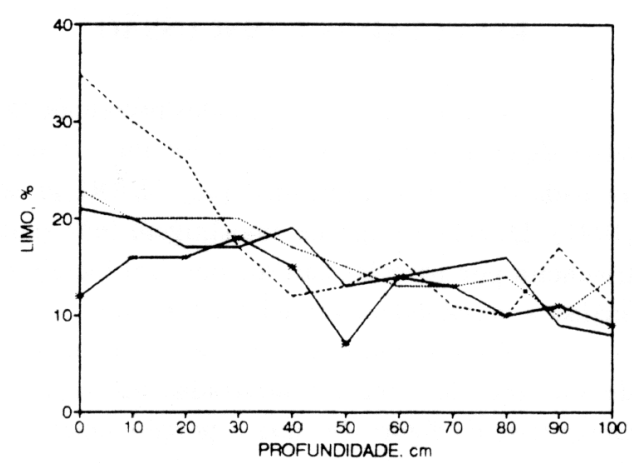

c)

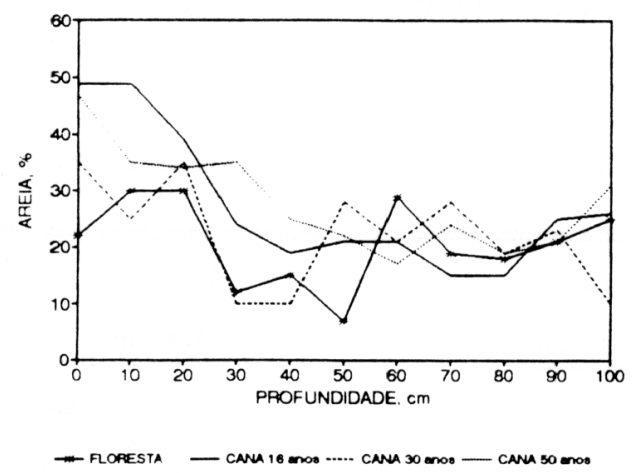

Figura 1 - Porcentagem de argila (a), silte (b) e areia total (c) ao longo do perfil, para as quatro áreas estudadas. sendo:

$$
I_{z_{1}}^{z_{2}}=\int_{z_{1}}^{z_{2}} P(z) d z
$$

onde $\mathrm{P}$ é a porcentagem das respectivas frações de tamanho de partículas, $\mathrm{z}$ a profundidade do solo, podem ser obtidos os valores médios das frações granulométricas no perfil (figuras $1 \mathrm{a}, 1 \mathrm{~b}$ e $1 \mathrm{c}$ ). Os cálculos foram feitos para o perfil total $\mathrm{z} 1=0 \mathrm{a}$ $\mathrm{z2}=100 \mathrm{~cm}$ e para duas camadas, $\mathrm{z} 1=0 \mathrm{a} \mathrm{z2}=$ $60 \mathrm{~cm} \mathrm{e} \mathrm{z} 1=60$ a z2 $=100 \mathrm{~cm}$ (TABELA 1 ). $\mathrm{O}$ limite de $60 \mathrm{~cm}$ foi escolhido, pois, as grandes variações no manejo agrícola do solo ocorrem nos primeiros $60 \mathrm{~cm}$ de profundidade.

Observa-se, desses resultados, que a quantidade acumulada de argila em todo o perfil varia de $65 \%$ (floresta) para 58,59 e $57 \%$, respectivamente, para as áreas de 16,30 e 50 anos de cultivo. No entanto, nota-se que, de 60 a $100 \mathrm{~cm}$, as quantidades acumuladas de argila mantiveram-se praticamente constantes, com valores de $65 \%$ no solo virgem e de 68,66 e $65 \%$ nos perfís cultivado por 16,30 e 50 anos, respectivamente, variações estas que podem ser atribuidas a variabilidade espacial natural do solo. Já entre a superfície e 60 $\mathrm{cm}$, as quantidades acumuladas de argila variaram mais acentuadamente: de $64 \%$ (floresta) para 57 , 55 e $51 \%$, respectivamente, nas áreas cultivadas. Com relação às quantidades de silte e areia, aparentemente não houve mudanças significativas. Os pequenos aumentos e decréscimos podem ser consequência das variações percentuais das quantidades de argila. Estes resultados sugerem perdas de argila ao longo do tempo, provavelmente por erosão.

$\mathrm{Na}$ figura 2 são apresentados os perfis de densidade do solo obtidos nas quatro áreas estudadas. Nota-se na figura $2 a$ que, na área sob floresta, a densidade do solo na região superficial foi bastante baixa, devido ao acúmulo de raízes e material orgânico em decomposição. Comparandose os perfis das áreas cultivadas com o da floresta, nota-se existir uma diferença de densidade bastante acentuada na região superficial e uma diferença menos acentuada nas regióes mais profundas. 
a)
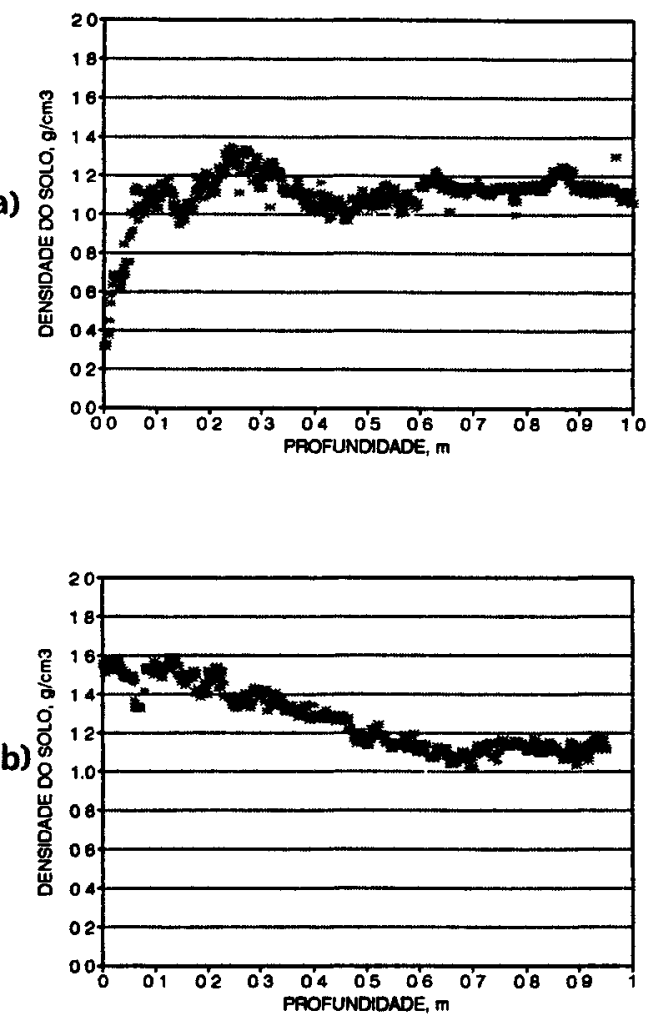
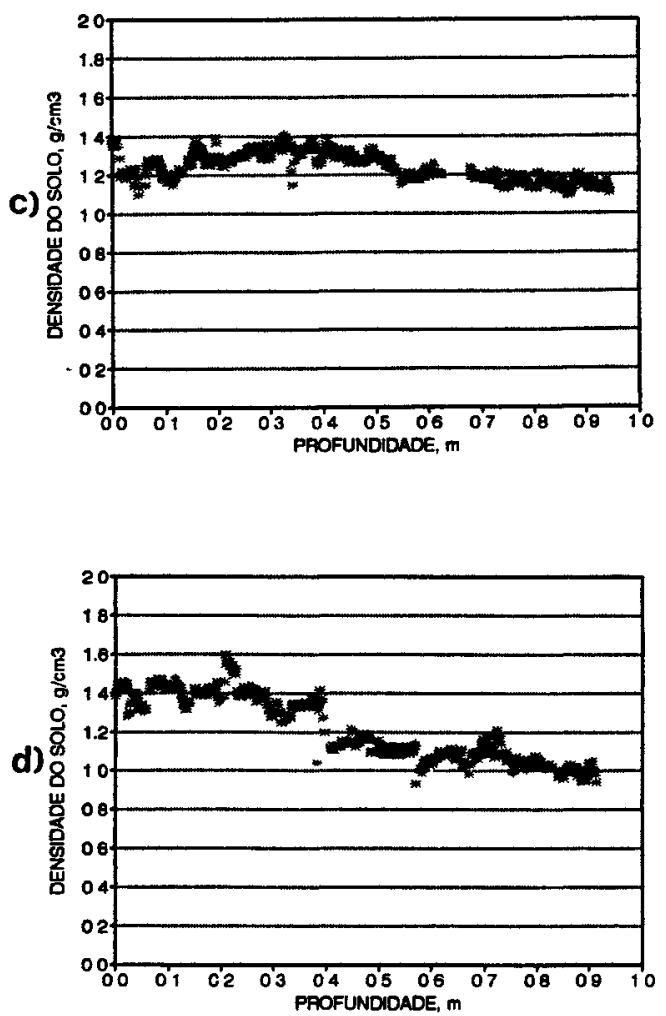

Figura 2 - Perfil de densidade, obtido dos torrões de solo, para área de floresta (a), cana-de açúcar por 16 anos (b), 30 anos (c) e 50 anos (d).

Aparentemente, a área de 16 anos apresenta uma maior compactação que as de 30 e 50 anos. Para melhor quantificar a compactação ao longo do perfil, calculou-se a integral:

$$
I_{z_{1}}^{z_{2}}=\int_{z_{1}}^{z_{2}} D s_{(z)} d_{(z)}
$$

onde Ds é a densidade do $\operatorname{solo}\left(\mathrm{g} / \mathrm{cm}^{3}\right)$. Esses resultados são apresentados na TABELA 2.

Pode-se observar que, até a profundidade de $60 \mathrm{~cm}$, os perfis de densidade das áreas de 16 , 30 e 50 anos apresentaram aumentos de 20,13 e $14 \%$, respectivamente, quando comparados à área sob floresta. A possível causa destas pequenas diferenças em valores de densidade, para as áreas de 30 e 50 anos pode ser explicada pelo nível de mecanização da cultura que há 30 anos e, mais ainda, há 50 anos atrás, era muito pequeno e, portanto, a agressão ao solo, muito menor. Assim, aparentemente, os solos que passaram por uma fase inicial de manejo menos agressiva apresentaram compactações menores ao longo do perfil do que aqueles que desde $o$ início foram manejados com um alto grau de mecanização. Observa-se também que, a partir de aproximadamente $50 \mathrm{~cm}$ de profundidade, os perfis de densidade possuem valores razoavelmente constantes $e$ idênticos para todas as áreas (em torno de $1,1 \mathrm{~g} / \mathrm{cm}^{3}$ ). 
a)

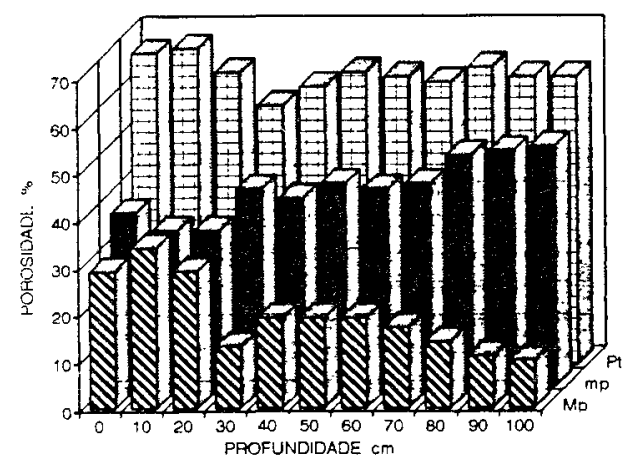

b)

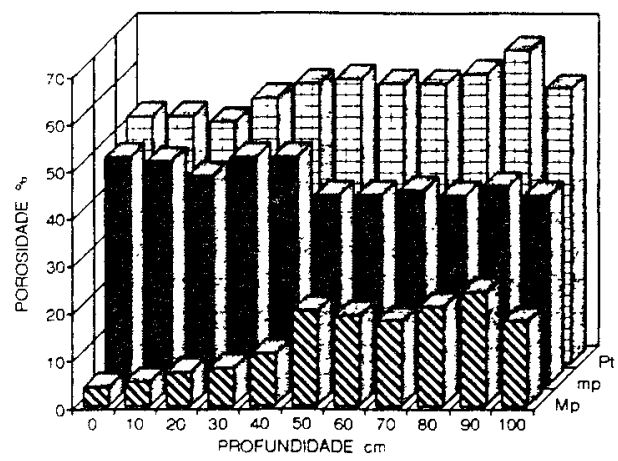

c)

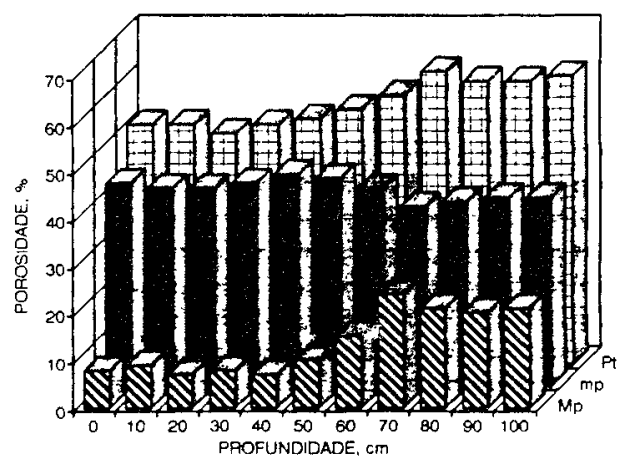

d)

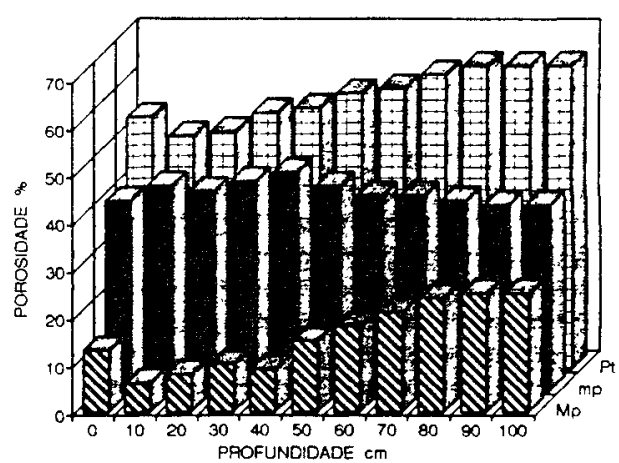

Figura 3 - Distribuição de macro (Mp), micro (mp) e porosidade total (Pt), em função da profundidade, para a área de floresta (a), cana-de-açúcar por 16 anos (b), 30 anos (c) e 50 anos (d).

TABELA 2 - Integral dos valores de densidade no perfil, no intervalo de 0 a $60 \mathrm{~cm}$ de profundidade, para as quatro áreas estudadas.

\begin{tabular}{ccc}
\hline Área & $\begin{array}{c}\mathrm{I} \\
\left(\mathrm{g} / \mathrm{cm}^{2}\right)\end{array}$ & $\mathrm{I} / \mathrm{I}_{\circ}$ \\
\hline Floresta & 67,44 & 1,00 \\
cana 16 anos & 80,94 & 1,20 \\
cana 30 anos & 76,03 & 1,13 \\
cana 50 anos & 76,87 & 1,14 \\
\hline
\end{tabular}

Como era esperado, em relação aos resultados de porosidade, observa-se, na Figura 3, que a porosidade total na área de floresta é maior em relação às outras áreas estudadas, como consequência da densidade menor. Da mesma forma para as áreas de 16, 30 e 50 anos de plantio de cana-de-açúcar pode-se observar que os valores de porosidade total diminuem, principalmente, na camada de $0-30 \mathrm{~cm}$.

KIEHL (1979) assume como solo ideal aquele que contém $1 / 3$ de macroporos e $2 / 3$ de microporos e THOMASSON (1978) classifica solos de acordo com sua estrutura, mostrando que um solo é tanto melhor quanto mais aerado, embora contendo boa quantidade de àgua disponível. A este respeito a área de floresta é a que mais se aproxima da situação ideal de um solo (TABELAS 3 e 4). Já para as áreas de 16,30 e 50 anos de plantio de cana-de-açúcar há excesso de microporos $e$, somente nas camadas mais profundas a proporção de macro e microporos aproxima-se da ideal. 
TABELA 3 - Porcentagens de macroporos (Mp) em relação à porosidade total $(\mathrm{Pt})$, nos perfis de floresta e cana-de-açúcar com 16, 30 e 50 anos de plantio.

\begin{tabular}{ccccc}
\hline $\begin{array}{c}\text { Profun- } \\
\text { didade } \\
\mathrm{cm}\end{array}$ & \multicolumn{5}{c}{ Mp/Pt (\%) } \\
\cline { 2 - 5 } & Floresta & 16 anos & 30 anos & 50 anos \\
\hline 0 & 44 & 8 & 16 & 25 \\
10 & 51 & 10 & 18 & 12 \\
20 & 47 & 14 & 14 & 16 \\
30 & 24 & 14 & 16 & 19 \\
40 & 32 & 19 & 13 & 16 \\
50 & 31 & 33 & 19 & 26 \\
60 & 31 & 32 & 26 & 31 \\
70 & 28 & 31 & 39 & 34 \\
80 & 22 & 34 & 35 & 38 \\
90 & 18 & 36 & 33 & 39 \\
100 & 17 & 31 & 34 & 39 \\
\hline
\end{tabular}

TABELA 4 - Porcentagens de macroporos (Mp) em relação à porosidade total $(\mathrm{Pt})$, nos perfis de floresta e cana-de-açúcar com 16, 30 e 50 anos de plantio.

\begin{tabular}{ccccc}
\hline $\begin{array}{l}\text { Profun- } \\
\text { didade } \\
\mathrm{cm}\end{array}$ & \multicolumn{4}{c}{ Mp/Pt (\%) } \\
\cline { 2 - 5 } & Floresta & 16 anos & 30 anos & 50 anos \\
\hline 0 & 56 & 92 & 84 & 75 \\
10 & 49 & 90 & 82 & 88 \\
20 & 53 & 86 & 86 & 84 \\
30 & 76 & 86 & 84 & 81 \\
40 & 68 & 81 & 87 & 84 \\
50 & 69 & 67 & 81 & 74 \\
60 & 69 & 68 & 74 & 69 \\
70 & 72 & 69 & 61 & 66 \\
80 & 78 & 66 & 65 & 63 \\
90 & 82 & 64 & 67 & 61 \\
100 & 87 & 69 & 66 & 61 \\
\hline
\end{tabular}

\section{CONCLUSÕES}

Houve variação significativa das características físicas das camadas superficiais do solo submetido a cultivo prolongado de cana-de-açúcar. Esta variação é função do tempo de cultivo, quando analisadas as diminuições na fração argila. Quanto à compactação e porosidade, o efeito maior foi na área de 16 anos de plantio, que foi desde o início manejada com um alto grau de mecanização.

Compactação e porosidade foram mais afetadas pelo manejo do que pelo tempo de cultivo. Ao contrário, as possíveis modificações nos teores de argila parecem depender mais do tempo de cultivo do que das formas de manejo.

\section{REFERÊNCIAS BIBLIOGRÁFICAS}

BORGES, A.L. Alteração das propriedades de um latossolo amarelo de Cruz das Almas, Bahia, pelo cultivo com frutíferas perenes e mandioca. Piracicaba, 1993. 161p. Tese (Doutorado) - Escola Superior de Agricultura "Luiz de Queiroz", Universidade de São Paulo.

CERRI, C.C.; FELLER, C.; CHAUVEL, A. Evolução das propriedades de um latossolo vermelho escuro após desmatamento e cultivo por doze e cinquenta anos com cana-de-açúcar. Cah. ORSTOM, série Pedologica, v.26, n.1, p.37-50, 1991.

CINTRA, F.L.D.; CUNHA, G.A.P. Caracterizaçāo física de solos cultivados com abacaxi em áreas com diferentes tempos de utilização. Revista Brasileira de Fruticultura, Cruz das Almas, v.9, n.3, p.7-15, 1987.

CRESTANA, S.; CRUVINEL, P.E.; VAZ, C.M.P.; CESAREO, R.; MASCARENHAS, S.; REICHARDT, K. Calibração e uso de um tomógrafo computadorizado em ciência do solo. Revista Brasileira de Ciência do Solo, Campinas, v.16, n.2, p.161-167, 1992.

KIEHL, E.J. Manual de edafologia-relações solo-planta. São Paulo, Agronômica Ceres, 264p. 1979.

OLIVEIRA, J.C.M. Atenuação de raios gama na análise granulométrica de amostras de terra. Piracicaba, 1994. 67p. Tese (Doutorado) - Centro de Energia Nuclear na Agricultura, Universidade de São Paulo.

SILVA, I.de F.da. Efeitos de sistemas de manejo e tempo de cultivo sobre propriedades fisicas de um Latossolo. Porto Alegre, 1980, 60p. Dissertação (Mestrado)

SILVA, M.S.L.; RIBEIRO, M.R. Influência do cultivo contínuo da cana-de-açúcar em propriedades morfológicas e físicas de solos argilosos de tabuleiro no Estado de Alagoas. Revista Brasileira de Ciência do Solo, Campinas, v.16, n.3, p.397-402, 1992.

THOMASSON, A.J. Towards an objective classification of soil struture. The Journal of Soil Science, Oxford, v. 29 , n.1, p.38-46, 1978 .

VAZ, C.M.P.; CRESTANA, S.; REICHARDT, K. Tomografia computadorizada na avaliação da compactação de solos. Revista Brasileira de Ciência do Solo, Campinas, v.16, n.2, p.153-159, 1992.

VAZ, C.M.P.; OLIVEIRA, J.C.M.de.; REICHARDT, K.; CRESTANA, S.; CRUVINEL, P.E.; BACCHI, O.O.S. Soil mechanical analysis through gamma ray attenuation. Soil Technology, Cremlingen, v.5, n.4, p.319-325, 1992.

WOOD, A.W. Soil degradation management under intensive sugar cane cultivation in North Queensland. Soil Use Management, Queensland, v.1, n.4, p.120$124,1985$.

Entregue para publicação em

Aceito para publicação em 12.07 .95 